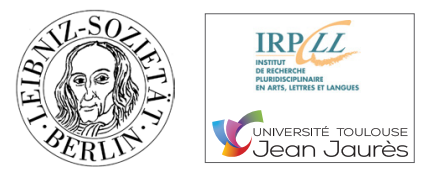

\title{
Kulturwissenschaft in den Philologien. Das Beispiel der deutschen Germanistik und Anglistik
}

\section{Susanne Greilich}

Universität Regensburg

\section{Abstract}

Nach langen Jahren der Debatten hat sich die Kulturwissenschaft in den philologischen Fächern in Deutschland etabliert. Sowohl hinsichtlich der programmatischen Auseinandersetzung als auch der wissenschaftlichen Praxis scheint eine Phase der vorläufigen Konsolidierung erreicht, die sich gleichwohl durch eine große Bandbreite im begrifflichen Verständnis und der konkreten Ausgestaltung von Kulturwissenschaft auszeichnet. Der Beitrag zeichnet diese Bandbreite auf der Grundlage von jüngeren Positionsbestimmungen und Einführungsbänden nach. Dabei gilt das Augenmerk den ,Nachbarphilologien' der Romanistik, spezifischer: der Germanistik und Anglistik.

After years of intense debate, Kulturwissenschaft has become firmly established in philology. It seems that programmatic positions as well as scientific practise have reached a phase of consolidation. Still, the notion of Kulturwissenschaft and its realization in philological research and teaching are broad, ranging from literary studies with a 'cultural' perspective to interdisciplinary Area Studies. The article gives an overview of this range, focussing on Romance studies' neighbouring disciplines, more precisely: the study of German and English.

Après des années d'intenses débats, la Kulturwissenschaft s'est établie dans les études consacrées aux langues, à la littérature et à la civilisation en Allemagne ; il semble que le stade de sa consolidation soit atteint. En revanche, les notions de Kulturwissenschaft ainsi que sa pratique scientifique - autant dans la recherche que dans l'enseignement universitaire - varient beaucoup : elles vont des Lettres jusqu'au cours de civilisation. L'article se propose de donner une vue d'ensemble de cette variété en se concentrant sur la germanistique et les études anglophones.

\section{Keywords}

Kulturwissenschaft $\bullet$ Cultural Studies $\bullet$ Germanistik $\cdot$ Anglistik $\bullet$ Status quo

Kulturwissenschaft $\bullet$ Cultural Studies $\bullet$ study of German $\bullet$ study of English $\bullet$ status quo

Kulturwissenschaft • Cultural Studies $•$ germanistique $\bullet$ études anglophones $\bullet$ état des lieux

Die Kulturwissenschaft behauptet sich - diese These oder besser: Beobachtung soll am Anfang des Beitrags stehen. Damit ist nun in erster Linie nicht die Idee gemeint, nach der die Kulturwissenschaft als neues Paradigma die philologischen Fächer derart auf den Kopf stellen würde, ja gar gestellt habe, dass die im Vergleich zu ihr ,alteingesessenen" Disziplinen der Literatur- und Sprachwissenschaften sich in ihrer bisherigen Gestalt bis hin zur Selbstverleugnung und -auflösung verändern würden. Von einer solchen ,Schreckensvision' scheinen zwar nicht wenige programmatische Aufsätze in der seit gut 25 Jahren schwelenden Debatte heimgesucht und sie hat sich u.a. in Form der Forderung nach einer ,Re-Philologisierung' (philological (re-)turn) der Fächer niedergeschlagen. Sie entspricht aber kaum der tatsächlichen Situation der Kulturwissenschaft innerhalb der Philologien. Um ,Behauptung' im Sinne von ,Beherrschung', von Dominanz soll es hier also nicht gehen.
Und die Feststellung der ,Behauptung der Kulturwissenschaft zielt umgekehrt auch nicht auf entsprechende, im Grunde rhetorische Verfahren der schlichten Etikettierung von Forschungsarbeiten und Lehrveranstaltungen als ,kulturwissenschaftlich", die aufgrund ihres Gegenstandsbereichs und ihrer Methodik vor dem cultural turn selbstverständlich (und korrekterweise) als der Literatur- oder der Sprachwissenschaft zugehörig betrachtet worden sind (vgl. Lüsebrink 2001: 72-73). Noch weniger zielt sie auf den im Lauf der Debatte wiederholt an die Kulturwissenschaft gerichteten Vorwurf der ,Pseudo-Disziplin“ und ,Halb-Wissenschaft', „die innerhalb des Wissenschaftsbetriebs eine ,Bühne' bereit [stelle], auf der nahezu jedes Thema auf nahezu jede Weise von nahezu jeder Person zur Aufführung gebracht werden könne" (Suhr / Wiechens 1998: 4). Auch um ,Behauptung' im Sinne einer ,fingierten Aussage' geht es hier nicht. Solcherlei Anwürfen, die 
insbesondere aus der Anfangszeit der Debatte um die Kulturwissenschaft datieren, ist bereits in einer ganzen Reihe von Artikeln und Vorworten widersprochen worden, wobei es bemerkenswerterweise scheint, als hätten gerade die genannten Anwürfe als movens einer stärkeren Konturierung der Kulturwissenschaft gewirkt.

Die Beobachtung, die hier am Anfang stehen soll, ist eine viel schlichtere. Sie zielt auf die Tatsache, dass die Kulturwissenschaft - auch in und unter Zutun der ,Nachbarphilologien' der Germanistik und Anglistik - ,von sich spricht'. Sie tut dies in einer Fülle von Publikationen, die von programmatischen Aufsätzen über Einführungs- und Lehrbücher für Studierende hin zu Handbüchern reichen; ${ }^{1}$ sie tut dies über die Namensgebung und Einrichtung spezifischer Studienangebote, die Umbenennung von Professuren, Lehrstühlen und Fakultäten. Sie spricht von sich mal im Singular und mal im Plural, mitunter auch gleichzeitig in beiden Formen: als Kulturwissenschaft(en) und sie versteht sich dabei wahlweise als Metaebene der Reflexion und Steuerungsebene für die Modernisierung der Geisteswissenschaften, als eigenständige Disziplin oder - zumeist - als transdisziplinär ausgerichtete Forschungspraxis (vgl. Fauser 2006: 9). Mit diesem ,Von-Sich-Sprechen' konstatiert die Kulturwissenschaft dabei einerseits ihre Existenz (auch) innerhalb der Philologien, sie ,manifestiert sich', und andererseits wird in diesem Sprechen das Verhältnis zu den etablierten Disziplinen und Fächern bestimmt, werden neue Perspektiven und Gegenstandsbereiche entwickelt.

Wenngleich Aleida Assmann - u.a. mit Blick auf die zu Beginn des Jahrtausends erfolgte Standortbestimmung der Kulturwissenschaft in der Anglistik (vgl. Klein / Kramer 2004) - in ihrer jüngst neu aufgelegten Einführung einerseits einen vorläufigen Schlussstrich unter die Diskussion um die Kulturwissenschaft zieht und andererseits angesichts neuer interdisziplinärer Forschungsfelder bereits die nächste Phase der Neuorientierung des Fachs ausruft (vgl. dies. 2017: 5), so kann es im Kontext der Frage nach der Profilierung der Kulturwissenschaft in der Frankoromanistik gleichwohl interessant und ggf. hilfreich sein, einen Blick auf den Status quo der Kulturwissenschaft in den benachbarten Fächern der Anglistik und der Germanistik zu werfen. Dies erscheint auch deshalb relevant, weil - wie Nünning / Nünning treffend bemerken - das interdisziplinäre Diskussionsfeld der Kulturwissenschaft(en) inzwischen auch für Fachleute kaum noch überschaubar ist (vgl. dies. 2008: XII). Dies gilt 2018 noch mindestens genauso wie 10 Jahre zuvor - und dies gilt u.U. auch für das Wissen der Frankoromanistik um die Kulturwissenschaft in den Nachbarphilologien.

Zu diesem Aspekt tritt der des interdisziplinären Impulses hinzu, die Frage nach möglichen Übertragungen und Kooperationen über die Grenzen des Fachs hinweg. In ihrem

1 Aus einer langen Liste von Veröffentlichungen aus der Germanistik und Anglistik (bzw. unter Mitbeteiligung von Germanisten und Anglisten) seien an dieser Stelle als Auswah genannt: Assmann 2017; Därmann / Jamme 2007; Fauser 2006; Joachimsthaler / Kotte 2008; Müller-Funk 2008; Nünning 2005; Nünning / Nünning 2008; Nünning / Sommer 2004 Rosenberg / Stratman 2008; Schößler 2006; sowie die aktualisierten Neuauflagen vo Bachmann-Medick 2004 und Hansen 2011. Zu den vollständigen Angaben vgl. die Bibliographie am Ende dieses Artikels.
Band Kulturwissenschaftliche Literaturwissenschaft konstatieren Nünning / Sommer den „theoretischen, methodischen und bildungspolitischen Schulterschluss“ (dies. 2004: 9) sowohl der Gegner als auch der Befürworter einer kulturwissenschaftlichen Erweiterung der Literaturwissenschaft in der Germanistik, Anglistik und Romanistik:

Verläßt man [...] kurz die nur zu vertrauten Diskussionen innerhalb des eigenen Faches, um einmal an den literaturwissenschaftlichen Türen der philologischen Nachbardisziplinen zu lauschen, so wird man mit einigem Erstaunen feststellen können, daß sich die Argumente [...] zum Verwechseln ähneln. (ebd.)

In der aus diesen Parallelismen resultierenden Möglichkeit einer fächerübergreifenden Debatte über die Stellung der Kulturwissenschaft innerhalb der Philologien, über ihre „Theoriegebäude, Argumentationsstränge und Erkenntnisinteressen“ (ebd.: 10), über die Fragen nach Anschluss an bzw. Abgrenzung von den Sozial- und Geschichtswissenschaften und der Landeskunde, erkennen die Autoren ein spezifisches Potenzial. Dieses Potenzial scheint nun nicht allein den oben skizzierten Gemeinsamkeiten der Fachdiskussionen geschuldet, als vielmehr auch und im Besonderen den Differenzen, oder genauer: dem Umstand, dass die Fächer ihre je eigenen fachspezifischen Traditionen und kulturwissenschaftlichen Vorläufer zusätzlich zu unterschiedlichen Positionen in die Debatte eingebracht haben.

Der vorliegende Beitrag greift die interdisziplinäre Zusammenschau - die, wenn man so will, regards croisés der philologischen Fächer -, die Nünning / Sommer ihrem Konzept einer kulturwissenschaftlichen Literaturwissenschaft zugrunde legen, auf, tritt aber zugleich einen Schritt zurück, um die Gesamtheit möglicher "Schulterschlüsse“ der quer durch die Philologien sich manifestierenden Positionen in den Blick zu bekommen. Denn die Beschreibung einer kulturwissenschaftlichen Literaturwissenschaft, wie sie Nünning / Sommer unter Beteiligung namhafter Kolleg_innen aus der Anglistik, Germanistik und Romanistik vornehmen, ist nicht nur Erhebung des Stands transdisziplinärer wissenschaftlicher Praxis, sondern ebenso sehr Programmatik; eine letztlich auch ideologische Verortung in Hinblick auf die Frage nach dem Verhältnis der philologischen Fächer und Disziplinen zum kulturwissenschaftlichen Paradigma bzw. der Idee davon, was Kulturwissenschaft sei. Selbstverständlich sind andere Positionsbestimmungen, Programmatiken und Konzepte einer philologischen Kulturwissenschaft bzw. der Kulturwissenschaft(en) in den Philologien denkbar und auch vorhanden.

Einen Eindruck von der Bandbreite dieser Vorstellungen vermittelt die von Ansgar und Vera Nünning herausgegebene Einführung in die Kulturwissenschaften von 2008. In diesem Band werden nicht nur theoretische Grundlagen und Schlüsselbegriffe des interdisziplinären Diskussionsfelds Kulturwissenschaften vorgestellt, sondern auch verschiedene Konzepte davon präsentiert, wie Kulturwissenschaft in der akademischen Praxis der Philologien, in Lehrangebot 
und Studienganggestaltung in Deutschland gefasst ist bzw. werden könnte: beispielsweise als Fremdkulturwissenschaft in Form interdisziplinärer Studien zu einem spezifischen Kulturraum (Area Studies, Landes- und Regionalwissenschaften) oder auch in grenzüberschreitender, interkultureller Perspektive (Interkulturelle Kommunikation), als interdisziplinäre Fremdheitsforschung (Kulturwissenschaftliche Xenologie), als Medienkulturwissenschaft oder eben als kulturwissenschaftlich orientierte Literaturwissenschaft.

Vor diesem Hintergrund soll konkreter danach gefragt werden, wie sich die Kulturwissenschaft in den philologischen Nachbardisziplinen der Anglistik und Germanistik in Deutschland behauptet, wie sie sich in Hinblick auf die Literatur- und ggf. die Sprachwissenschaft verortet, welche Zugriffe sie privilegiert, welche thematischen Schwerpunkte sie setzt. Es soll weder darum gehen, die Genealogie der deutschsprachigen Kulturwissenschaften einerseits und die der angelsächsischen Cultural Studies andererseits noch einmal nachzuzeichnen, noch die Rezeption der Cultural Studies in der deutschen Wissenschaftslandschaft vorzustellen. ${ }^{2}$ Das Augenmerk soll vielmehr der aktuellen Verortung und Gestalt der Kulturwissenschaft in der deutschen Anglistik und Germanistik gelten. Dabei erscheint es interessant, sich nicht allein entsprechenden programmatischen Aufsätzen zu widmen, sondern auch und im Besonderen den in den letzten Jahren herausgegebenen, vielfach neu aufgelegten Einführungsbänden zum Thema. Sie sind in besonderer Weise nicht nur akademische Standortbestimmung, sondern sie fördern und dokumentieren auch die Durchsetzung bestimmter Vorstellungen und Praktiken des kulturwissenschaftlichen Arbeitens. Damit sind sie Ausdruck sowohl des diskursiven wie auch des im weiteren Sinne institutionellen Status quo der Kulturwissenschaft in der Germanistik und Anglistik.

Auf eine Übersicht der kulturwissenschaftlichen Studienangebote bzw. Graduiertenprogramme unter Federführung bzw. Beteiligung der deutschen und englischen Philologie muss an dieser Stelle verzichtet werden, verwiesen sei hier auf die - freilich schon etwas in die Jahre gekommenen - Übersichten bei Böhme / Matussek / Müller (dies. 2002: 210-211) bzw. Teil 1 der ,Standortbestimmung' der Anglistik von 2003, in der u.a. das Gießener Graduiertenzentrum für die Erforschung von Kultur (ehemals: Gießener Graduiertenzentrum Kulturwissenschaften) mit seinen Studien- und Qualifikationsprogrammen, der Passauer „Kulturwirt“ und - exemplarisch - das Großbritannienzentrum (Centre for British Studies) der Humboldt-Universität vorgestellt werden (vgl. Klein / Kramer 2004: 1-13). ${ }^{3}$

2 Zur Rezeption der Cultural Studies in der Germanistik unter Rückbesinnung auf die Traditionen der deutschen Kulturwissenschaften um 1900 einerseits und die Rolle der ostdeutschen Kulturwissenschaft bzw der Kritischen Theorie in diesem Kontext andererseits vgl. Herrmann 2004: 39-43.

3 Mindestens eines der vorgestellten Studienangebote hat in der Zwischenzeit seine Gültigkeit verloren. Das Tübingen Centre for Interdisciplinary British Studies (TCIBS), das sich bereits 2003 erklärtermaßen in einer durchaus kritischen Interimsphase (TCl sich bereits 2003 erklärtermaßen in einer durchaus kritischen Interimsphase befand, ist ofenbar nicht weitergeführt worden; das Englische Seminar der Universität weist nun einen auserich o orming. Zum Gießener Graduiettenzentr html; zum Centre for British Studies: www.gbz.hu-berlin.de; zum Passauer Kulturwirt: www. uni-passau.de/bachelor-kulturwirtschaft.
Eine Zusammenschau entsprechender Publikationen der letzten Jahre, die die ,Kulturwissenschaft/en“ oder das Adjektiv ,kulturwissenschaftlich' im Titel tragen, lässt mit Blick auf die Frage nach dem derzeitigen Ort der Kulturwissenschaft in den Philologien bzw. ihrer Bedeutung für die philologischen Fächer zwei große Tendenzen erkennen, die sich zumeist bereits in der Wahl des im Titel verwendeten Terminus ,Kulturwissenschaft', (im Singular) bzw. ,Kulturwissenschaften", (im Plural) manifestieren.

1) So ist, genau wie in anderen Geistes- bzw. den Sozialund Gesellschaftswissenschaften, eine deutliche Tendenz erkennbar, dort im Singular von Kulturwissenschaft zu sprechen, wo sich eine etablierte Disziplin in ihrem Verhältnis zum kulturwissenschaftlichen Paradigma verortet, ergo: sich selbst ,als (eine) Kulturwissenschaft' bestimmt, ${ }^{4}$ wodurch sich in Summe ein multiples Gebilde von Kulturwissenschaften im Sinne einer Perspektivierung vieler einzelner Disziplinen als Kulturwissenschaften ergibt.

Dies gilt im Bereich der philologischen Fächer insbesondere für die Literaturwissenschaft, mit der Folge, dass auch in der Germanistik die Kulturwissenschaft in enger Verbindung mit der Literaturwissenschaft begriffen bzw. betrieben wird. ${ }^{5}$ Ein solcher Nexus findet sich durchaus auch in der Anglistik das o.g. ,Studienbuch“ von Nünning / Sommer (dies. 2004) verweist in aller Deutlichkeit auf die Transdisziplinarität des Phänomens -, wenngleich sich die Kulturwissenschaft hier in Form entsprechender, interdisziplinär angelegter Studienangebote (als British Studies, British Cultural Studies) zugleich als ,dritte Säule' neben der Literatur- und Sprachwissenschaft etabliert hat. Auf diesen Aspekt wird zurückzukommen sein.

Die Germanistin Britta Herrmann verweist in einem Aufsatz auf die enge Verbindung zwischen Literaturwissenschaft und Kulturwissenschaft bereits bei den ,Gründervätern' des Birmingham Center of Contemporary Cultural Studies, Richard Hoggart und Raymond Williams (vgl. dies. 2004: 44-45). Sie macht den Nexus dabei an der Methode der beiden Literaturwissenschaftler fest, auch andere kulturelle Erscheinungsformen als die der Literatur wie einen Text zu lesen. Auch die Ethnologie (C. Geertz) und die Geschichtswissenschaft $(\mathrm{H}$. White) haben in und mit ihren Arbeiten die narrative Verfasstheit von Kultur hervorgehoben. Die ethnographische Metapher von der „Kultur als Text" ist - so Herrmann weiter - vom New Historicism in Richtung der Literatur zurückgewendet worden, Kultur wurde „nun nicht nur als, sondern im (poetischen) Text analysiert" (ebd.: 45).

Während Wolfgang Müller-Funk den skizzierten narratologischen Ansatz der Cultural Studies vor einiger Zeit zum Ausgangspunkt einer narrativen Theorie der Kulturwissenschaften gemacht und dabei zuletzt den Zusammenhang

\footnotetext{
4 Die genannte Terminologie findet in einer Vielzahl von Überlegungen anderer, auch ,kleinerer' Fächer zum Thema, wie etwa der Vergleichenden Religionswissenschaft, de Musikwissenschaft, der Indologie, etc. bis hin zur Kulturpsychologie und Erziehungswissenschaft. Vgl. dazu beispielsweise die Aufsätze in Conermann 2012 und Müller 2003.

5 Auf diese auch in der Frankoromanistik bestehende enge Verbindung und die damit verbundene Problematik hat Dorothee Röseberg jüngst verwiesen (Röseberg: 2018).
} 
zwischen Narrativität und Gedächtnis sowie Narration, Identität und Geschlechterkonstruktion in den Mittelpunkt gestellt hat (vgl. ders. 2008) und Doris Bachmann-Medick die Metapher von der „Kultur als Text“ nutzt, um vor dem Hintergrund einer Verschränkung von Literaturwissenschaft und Ethnologie die Forschungsrichtung einer literarischen Kulturanthropologie zu begründen (vgl. dies. 2004), fokussiert Herrmann in ihrem Artikel auf den Mechanismus der Literaturwissenschaft, ihre Definition als Kulturwissenschaft im Wesentlichen aus den o.g. Zusammenhängen zwischen Kultur und Literatur zu ziehen. Mit Blick auf Greenblatts Theorie einer Kulturpoetik schreibt sie:

Dadurch werden LiteraturwissenschaftlerInnen gleichsam per se und auf ihrem ureigensten [sic] Gebiet zu KulturwissenschaftlernInnen [sic]. Indem Greenblatt der symbolischen Dimension kulturellen Handelns in den kanonischen Texten nachspürt und dabei etwa untersucht, wie Macht mit Hilfe von Bedeutungen ausgehandelt wird [...], verwandelt sich der ursprünglich politische Impetus der marxistisch inspirierten British Cultural Studies in Semiotik: die Zirkulation sozialer Energien erscheint als Zirkulation der Zeichen.

Damit ist der cultural turn auch wieder in der Literaturwissenschaft angekommen, von der er als interpretative turn zunächst seinen Ausgang genommen hat. Kultur als Text zu verstehen, dürfte gerade für die deutsche Literaturwissenschaft deshalb so attraktiv geworden sein, weil sich hier eine neue Legitimation für das Fach Germanistik abzeichnet: Sind nicht gerade seine VertreterInnen prädestiniert für semiotische und poetische Kulturforschungen? Können nicht gerade sie in Zeiten vielfacher Umbrüche (Wende, Migrationsbewegungen, postnationale Konstellationen) Fragen nach kulturellen Identitäten oder Differenzen und nach Formen des kulturellen Gedächtnisses aus den in der Literatur gleichsam archivierten Wissensbeständen heraus beantworten?

Als ehemalige Nationalphilologie und Literatur-Wissenschaft scheint die Germanistik in Zeiten der Globalisierung und nach dem vermeintlichen Ende der Gutenberg-Galaxis ihren Kredit verloren zu haben. Als Kulturwissenschaft hätte sie, so scheint es, plötzlich die Chance, noch einmal jenen Platz in der Wissenschaftslandschaft zugewiesen zu bekommen, auf den sie im 19. Jahrhundert so stolz war und den sie heute nur noch aufgrund der stetig steigenden Studentenzahlen einnimmt. (Herrmann 2004: 45)

Über den Zirkelschluss Literaturwissenschaft $\rightarrow$ Kultur als Text (Ethnologie) $\rightarrow$ Kultur im Text (New Historicism) wird die Literaturwissenschaft letztlich nicht nur ab ovo als Kulturwissenschaft bestimmt, sondern mehr noch: literaturwissenschaftliches Arbeiten zur Kernkompetenz des kulturwissenschaftlichen Arbeitens erklärt. ${ }^{6}$

6 Es wäre zu fragen, ob diese selbstbewusste Behauptung von der Literaturwissenscha als einer ,Kulturwissenschaft per se' nicht zumindest mitverantwortlich ist für die Konturlosigkeit des kulturwissenschaftlichen Lehrangebots an manchen deutschen Instituten, wie sie Lüsebrink konstatiert (vgl. ders. 2001: 72).
Wer sich, um die Eingangsbemerkung aufzunehmen, in den entsprechenden, einschlägigen Einführungen in „die Kulturwissenschaft" der Anglistik und Germanistik behauptet, wer hier ,von sich spricht', ist folglich - vor dem Hintergrund des oben Gesagten kaum überraschend - die Literaturwissenschaft, die sich in Hinblick auf das kulturwissenschaftliche Paradigma legitimiert und partiell neu justiert. Der oben skizzierte Argumentationszusammenhang von der Literatur- als einer Kulturwissenschaft per se und der daraus im Umkehrschluss (Kulturwissenschaft als Literatur- bzw. Textwissenschaft) abgeleitete Anspruch der Literaturwissenschaft auf Theorieexport in angrenzende Fächer liegen auch Markus Fausers bereits in 3. Auflage erschienenen Einführung in die Kulturwissenschaft (Darmstadt, WBG, 2004) zugrunde, die auf zentrale kulturwissenschaftliche Literaturtheorien (literarische Anthropologie, Handlungs- und Wahrnehmungstheorien, Gender Studies, Gedächtnistheorien, Intertextualität) fokussiert.

In der Formulierung der Literaturwissenschaft ,als Kulturwissenschaft" wird andererseits das Bestreben erkennbar, die Disziplin unter Berücksichtigung kulturwissenschaftlicher Fragestellungen zu ,modernisieren', indem kulturwissenschaftliche Perspektiven implementiert werden. Die Germanistin Franziska Schößler umreißt diese Vorstellung in ihrem Band Literaturwissenschaft als Kulturwissenschaft (2006) mit den folgenden Worten:

Aus der Perspektive der Philologien zeichnet sich die Tendenz ab, kulturwissenschaftliche Themenstellungen zu integrieren und mit bestehenden methodologischen Verfahren zu vernetzen; gefordert wird eine literaturwissenschaftliche Kulturwissenschaft, die das Profil der Disziplinen berücksichtigt und die ausdifferenzierten Lektürepraktiken nicht verspielt. [...] Der vorliegende Band möchte an die Tendenz anschließen, kulturwissenschaftliche Positionen in die Fächer zu integrieren und das methodologische Potential der kulturwissenschaftlichen Theoreme für die literaturwissenschaftliche Analyse auszuloten. (Schößler 2006: VII-X; Herv. d. Verf.)

Bemerkenswert erscheint in diesem Zusammenhang die Deutlichkeit, mit der - in diesem wie in anderen entsprechenden Einführungsbänden und Texten - nicht nur auf die spezifische Berücksichtigung der Ästhetik literarischer Texte insistiert wird, sondern letztlich auch auf die Beschränkung des für die Literaturwissenschaft in Frage kommenden Korpus. ${ }^{7}$ Mit Blick auf das von Böhme / Scherpe 1996 evozierte Potenzial der Kulturwissenschaft als Medium interdisziplinärer Verständigung einerseits und dem von Frühwald u.a. 1991 erhobenen Befund einer drohenden Zersplitterung der Wissenschaftslandschaft zu Beginn der 1990er Jahre andererseits bemerkt die Verfasserin:

Interdisziplinarität gilt mithin als Chance und Gefährdung, und zwar auch deshalb, weil der kulturwissenschaftliche Fokus,

$\begin{array}{lll}7 & \text { Dieses Postulat spiegelt sich auch in der Auswahl der Texte streichen, auf die die } \\ \text { Autorin ihre kulturwissenschaftlichen Beispiellektüren appliziert. Sie sind alle dem hochkul- }\end{array}$ Autorin ihre kulturwissenscha 
der den literarischen Text tendenziell kontextualisiert, [...] eine Deprivilegierung des Literarischen mit sich bringt. Auch nichtliterarische Texte gelten als Texte, werden als narrative, rhetorisch organisierte Konstrukte wahrgenommen, so dass der Sonderstatus der Literatur gefährdet scheint und die Gefahr des Dilettantismus droht. Werden die Kulturwissenschaften jedoch in die einzelnen Disziplinen integriert, bei behutsamer Öffnung zu anderen Fächern, so ließe sich die Angst vor der puren „Liebhaberei“, vor der Unwissenschaftlichkeit sicher verringern. (ebd.: VIII)

In dieser Einlassung spiegelt sich zweifellos die in der Germanistik an der Jahrtausendwende geführte intensive Diskussion um die Risiken einer Literaturwissenschaft als Kulturwissenschaft, eine Diskussion, die stets von der Vision eines doppelten Definitionsverlusts der Literaturwissenschaft sowohl als Disziplin als auch in Hinblick auf ihre Gegenstände (schöngeistige Literatur des hochkulturellen Kanons) begleitet war. Gleichwohl scheint die Perspektive einer kulturwissenschaftlich orientierten Literaturwissenschaft mehrheitsfähig geworden zu sein. Auch Schößler betont das Potenzial, durch kulturwissenschaftliche Ansätze die Literatur (wieder) an soziale Prozesse zurückzubinden und durch den Anschluss an entsprechende theoretische Konzepte (Kultur ,als Gedächtnis‘, Kultur ,als Diskurssystem', Gender-Theorie, postkoloniale Theoriebildung, etc.) kulturelle Mechanismen des Ein- und Ausschlusses oder die Verknüpfung von kultureller Repräsentation mit Machtinteressen in den Fokus der literaturwissenschaftlichen Analyse zu bekommen.

In Richtung einer Neuorientierung der Literatur- als Kulturwissenschaft zielt auch die Anglistin Aleida Assmann mit ihrer mittlerweile in vierter Auflage erschienenen Einführung in die Kulturwissenschaft, wenngleich die Verfasserin weniger als etwa Schößler auf Kulturtheorien und ihre mögliche methodologische Nutzbarmachung für die literaturwissenschaftliche Analyse zielt, denn deutlicher - und aus der Sicht der adressierten studentischen Leserschaft insgesamt pragmatischer auf Themen und Fragestellungen der Kulturwissenschaft(en) fokussiert, die sie in insgesamt sieben Felder (Zeichen, Medien, Körper, Zeit, Raum, Gedächtnis, Identität) gruppiert. Es geht Assmann erklärtermaßen um die „Erforschung des Zusammenhangs von Literatur und wichtigen Grundfragen der Kultur" (Assmann 2017: 12); die Autorin begreift ihren Band zugleich als Einführung in die Kulturwissenschaft und in die englische und amerikanische Literatur unterschiedlicher Epochen.

Letztlich ergeben sich damit - sowohl durch die Berücksichtigung einschlägiger kulturwissenschaftlicher Theorien als auch die Fokussierung auf spezifische Themenbereiche - für die Philologien neue Möglichkeiten der (Re-)Lektüre literarischer Texte, allerdings ohne dass damit zwangsläufig eine Ausweitung der analysierten Gegenstände im Sinne eines erweiterten Literatur-, Medien- oder Textbegriffs verbunden wäre oder aber andere kulturelle Repräsentationssysteme als das der Literatur in den Blick genommen würden, die über eine u.U. notwendige ,Kontextualisierung“ hinausgingen. ${ }^{8}$

2) Neben der Selbstdefinition und partiellen Neuausrichtung der Literaturwissenschaft ,als (einer) Kulturwissenschaft' im oben skizzierten Sinne finden sich Publikationen, die die Kulturwissenschaft über die Bindung an eine je spezifische Disziplin (wie die der Literaturwissenschaft) hinaus betrachten oder anders gesagt, die sich an der gemeinsamen Formulierung übergreifender kulturwissenschaftlicher Perspektiven und Fragestellungen für das interdisziplinäre Forschungsfeld ,Kulturwissenschaft' beteiligen. Es geht den Herausgebern / Autoren dabei offensichtlich gerade nicht darum, „[...] dass jedes Fach seine eigene Kulturwissenschaft entwirft, eine spezifische Assemblage von kulturtheoretischen Konzepten zusammenstellt“" (Schößler 2006: VIII), sondern darum, Begriffe, Themen und Zugriffe zu formulieren, die sich für viele der sich als Kulturwissenschaft begreifenden Disziplinen (und damit für ,die Kulturwissenschaften') als anschlussfähig erweisen können. Solche Ansätze, die die Kulturwissenschaft aus der Pluralität der Fächer heraus in einer interdisziplinären Perspektive begreifen, betonen die Schnittmengen, die sich etwa in Hinblick auf Leitkategorien, Konzepte und Fragestellungen zwischen den einzelnen kulturwissenschaftlich orientierten Fächern ergeben (vgl. Jaeger 2003-2011). Als Schlüsselbegriffe und zentrale Themen lassen sich hier insbesondere Zeichen, Medien, Kommunikation, Identität, Alterität, Hybridität, Raum, Grenze, Zeit, Geschichte, Übersetzung, Transfer, Gedächtnis, Erinnern, Körper, Leiblichkeit festmachen (vgl. ebd., Bd. 1 und 3). ${ }^{9}$

3) Von diesen beiden Zugriffen auf die Kulturwissenschaft in den Philologien lässt sich schließlich ein dritter unterscheiden, der jenseits der starken Rückbindung der Kultur- an die Literaturwissenschaft andere Wege geht und Kulturwissenschaft innerhalb der Philologien in Form einer sogenannten ,dritten Säule' (neben Literatur- und Sprachwissenschaft) betreibt. In der deutschen Anglistik hat sich diese in Form der British Studies bzw. der British Cultural Studies etabliert, wenngleich deutliche Unterschiede zwischen den Regionen festzustellen sind. In ihrem Statusbericht von 2007 konstatieren von Rosenberg / Stratman, dass in den östlichen Bundesländern - vor dem Hintergrund der Tradition der Landeswissenschaft - ca. 80\% der anglistischen Studiengänge Cultural Studies als eine gleichberechtige Säule des Studiums behandeln, in denen des ehemaligen Westens der Republik allerdings nur etwa ein Drittel der Seminare (vgl. dies. 2007: 128). Zudem ist hier, so die Autoren, oftmals eine Form der Kulturwissenschaft / Cultural Studies vorzufinden, die sich „noch weitgehend im literaturwissenschaftlichen

8 Dass hinsichtlich der Gegenstände, die im Zuge einer kulturwissenschaftlichen Lite8 Dass hinsichtlich der Gegenstände, die im Zuge einer kulturwissenschaftlichen Litebestehen, zeigt auch ein Blick in den Band von Nünning / Sommer 2004.

9 Auch A. Assmann schließt - wiewohl es ihr um eine literaturwissenschaftliche Kulturwissenschaft geht - mit ihrer Einführung dezidiert an solche transdisziplinären Konzepte und Themen an. Es sei darauf verwiesen, dass die evozierten Schlüsselthemen und -begriffe zum Großteil und nicht zuletzt auch in den verschiedenen turns aufgehoben sind, die Bachmann-Medick in ihrem Band beschreibt (vgl. dies. 2014). 
Grenzgebiet bewegt" (ebd.: 129). Demgegenüber betonen Rosenberg / Stratman ein Konzept von Kulturwissenschaft in Form der Area Studies. Für diese sind zum einen vier Kulturbegriffe (Kultur als Konstruktion, Kultur als whole way of life, Kultur als Zeichensystem, Kultur als Gedächtnis) maßgeblich. Zum anderen machen die Area Studies wahlweise den interdisziplinären Zugriff auf einen Kulturraum stark, so dass neben Sprache und Literatur auch das politische, das Rechts- und Erziehungssystem, etc. in den Blick der Analyse geraten, (=British Studies / Kulturraumstudien) oder sie spezialisieren sich auf die Interpretation von Zeichenprozessen bzw. Repräsentationen, mit denen sich eine Gesellschaft über sich selbst verständigt (=British Cultural Studies). Hinsichtlich der Analyseobjekte stehen sowohl Sprache, Literatur, Kunst, Film und populäre Kultur im Zentrum, wie auch Rituale, Feste, etc. als signifying practices. In thematischer Hinsicht komme, so die Autoren, den Konstruktionsprozessen von Kultur (und Nation), kultureller Identität, Fremd- und Eigenbildern eine zentrale Rolle zu; in methodischer Hinsicht sei der Kulturvergleich hervorzuheben.

In der Germanistik findet sich ein landeswissenschaftlicher Zugriff auf ,die deutsche Kultur' allein in der Auslandsgermanistik; Fragen nach kultureller Verortung und Identitätskonstruktion werden (auch) durch die Forschungsrichtung der interkulturellen Germanistik behandelt.

Resümierend lässt sich konstatieren, dass die Kulturwissenschaft in den Nachbarphilologien der Anglistik und Germanistik eine deutliche Anbindung an die
Literaturwissenschaft gefunden hat. Die neuere deutsche Literaturwissenschaft hat mit kulturwissenschaftlichen Konzepten ihren Frieden gemacht, sie - auch angesichts eines möglichen, sowohl wissenschaftlichen wie gesellschaftlichen Bedeutungsaufschwungs - integriert und zur ,Modernisierung der Disziplin genutzt. Das „einvernehmlich[e] Nebeneinander unterschiedlicher Positionen von den Cultural Studies britischer Prägung mit stark landeskundlichem Einschlag bis hin zur kulturwissenschaftlich profilierten Literaturwissenschaft", das Nünning / Sommer mit Blick auf die deutsche Anglistik konstatiert haben (dies. 2004: 12), unterstreicht den Eindruck einer Konsolidierungsphase der Kulturwissenschaft auch in den Philologien. Nach einer langen Phase der programmatischen Debatte und Standortbestimmungen ist die Zeit der Zusammenschau interdisziplinär anschlussfähiger Schlüsselbegriffe und Themen gekommen (vgl. Jaeger et al. 2003-2011) bzw. der methodologischen Schärfung einzelner Kulturwissenschaften wie etwa der kulturwissenschaftlich orientierten Literaturwissenschaft (vgl. Nünning / Sommer 2004). Ob vor diesem Hintergrund auch für andere Notionen von Kulturwissenschaft (wie den Area Studies / Cultural Area Studies) ,Schulterschlüsse' und gekreuzte Blicke über die Grenzen des jeweiligen philologischen Fachs hinweg möglich und sinnvoll sind, wird zu prüfen sein. Unser ,Blick nach nebenan' soll an dieser Stelle aber enden. Es ist - so sei betont - der einer Frankoromanistin gewesen; es mag ihr manches entgangen und nicht ins Blickfeld geraten sein, dafür bittet sie um Nachsicht.

\section{Bibliographie}

Assmann, Aleida (2017), Einführung in die Kulturwissenschaft Grundbegriffe, Themen, Fragestellungen, 4., durchgesehene Auflage, Berlin, Erich Schmidt [Grundlagen der Anglistik und Amerikanistik, 27].

Bachmann-Medick, Doris (2014), Cultural Turns. Neuorientierungen in den Kulturwissenschaften, 6. Aufl., Reinbek bei Hamburg, Rowohlt.

Bachmann-Medick, Doris (Hg.) (2004), Kultur als Text. Die anthropologische Wende in der Literaturwissenschaft, 2., aktualisierte Auflage, Tübingen, A. Francke.

Böhme, Hartmut / Scherpe, Klaus R. (Hg.) (1996), Literatur und Kulturwissenschaften. Positionen, Theorien, Modelle, Reinbek bei Hamburg, Rowohlt.

Böhme, Hartmut / Matussek, Peter / Müller, Lothar (Hg.) (2002), Orientierung Kulturwissenschaft. Was sie kann, was sie will, 2. Aufl., Reinbek bei Hamburg, Rowohlt.

Conermann, Stephan (Hg.) (2012), Was ist Kulturwissenschaft? Zehn Antworten aus den „Kleinen Fächern“, Bielefeld, transcript.

Därmann, Iris / Jamme, Christoph (Hg.) (2007), Kulturwissenschaften. Konzepte, Theorien, Autoren, München, Wilhelm Fink.

Fauser, Markus (2006), Einführung in die Kulturwissenschaft, 3. Aufl., Darmstadt, WBG.

Frühwald, Wolfgang / Jauß, Hans Robert / Koselleck, Reinhart / Mittelstraß, Jürgen / Steinwachs, Burkhart (Hg.) (1991):
Geisteswissenschaften heute. Eine Denkschrift, Frankfurt a.M., Suhrkamp.

Hansen, Klaus P. (2011), Kultur und Kulturwissenschaft, 4., vollständig überarbeitete Auflage, Tübingen, A. Francke.

Herrmann, Britta (2004), „Cultural Studies in Deutschland: Chancen und Probleme transnationaler Theorie-Importe für die (deutsche) Literaturwissenschaft“, in Nünning, Ansgar / Sommer, Roy (Hg.), Kulturwissenschaftliche Literaturwissenschaft, Tübingen, Gunter Narr, 33-53.

Jaeger, Friedrich u.a. (Hg.) (2003-2011), Handbuch der Kulturwissenschaften, 3 Bd., Stuttgart, J.B. Metzler.

Klein, Bernhard / Kramer, Jürgen (Hg.) (2004), Kulturwissenschaften in der Anglistik. Eine Standortbestimmung. Dokumentation einer Tagung an der Universität Dortmund, 8. bis 10. April 2003, online-Publikation [http://www.britcult.de/doKomplett.pdf].

Joachimsthaler, Jürgen / Kotte, Eugen (Hg.) (2008), Kulturwissenschaft(en) in der Diskussion, München, Martin Meidenbauer.

Lüsebrink, Hans-Jürgen (2011), „Frankreichforschung und deutsch-französische Beziehungen in der (Franko-)Romanistik. Landeskunde/Kulturwissenschaft und Fremdsprachenphilologien“, in Grunewald, Michel u.a. (Hg.), France-Allemagne au XXe siècle - La production de savoir sur l'autre. Deutschland und Frankreich im 20. Jahrhundert-Akademische Wissensproduktion über das andere Land, Frankfurt a.M., Peter Lang, 69-74. 
Müller, Klaus E. (Hg.) (2003), Phänomen Kultur. Perspektiven und Aufgaben der Kulturwissenschaften, Bielefeld, transcript.

Müller-Funk, Wolfgang (2008), Die Kultur und ihre Narrative. Eine Einführung, 2. Aufl., Wien, Springer-Verlag.

Nünning, Ansgar (Hg.) (2005), Grundbegriffe der Kulturtheorie und Kulturwissenschaften, Stuttgart, J.B. Metzler.

Nünning, Ansgar / Nünning, Vera (Hg.) (2008), Einführung in die Kulturwissenschaften. Theoretische Grundlagen - Ansätze - Perspektiven, Stuttgart, J.B. Metzler.

Nünning, Ansgar / Sommer, Roy (Hg.) (2004), Kulturwissenschaftliche Literaturwissenschaft, Tübingen, Gunter Narr.

Röseberg, Dorothee (2018), „Kulturwissenschaft und Romanistik. Positionen und Verortungen“, in Ettrich, Jenny/ Mäder, Marie-
Therese (Hg.), Dialogpotenziale kulturwissenschaftlicher Forschung in den Fremdsprachenphilologien, Berlin, Peter Lang, 17-35.

Rosenberg, Ingrid von / Stratman, Gerd (2008), „Die Ankunft der Kulturwissenschaft in der Anglistik. Spaltung oder Chance?", in Schaefer, Ursula (Hg.), Der geteilte Gegenstand. Beiträge zur Geschichte, Gegenwart, und Zukunft der Philologie(n), Frankfurt a. M., Peter Lang, 127-138.

Schößler, Franziska (unter Mitarbeit von Bähr, Christine) (2006), Literaturwissenschaft als Kulturwissenschaft. Eine Einführung, Tübingen, A. Francke.

Suhr, André / Wiechens, Peter (1998), „Einführung“, in Düllo, Thomas / Berthold, Christian / Greis, Jutta / Wiechens, Peter (Hg.), Einführung in die Kulturwissenschaft, Münster, LIT, 3-17. 